\title{
Sarcoid vasculitis presenting with stroke: 3D T1 dark blood vessel wall imaging
}

Vasculite por sarcoidose associada a isquemia aguda: estudo da parede vascular pela técnica T1 3D "dark blood"

Pedro Neves Paiva de CASTRO', Roberto Queiroz dos SANTOS', Dequitier Carvalho MACHADO', Felipe da Rocha SCHMIDT², Denise Madeira MOREIRA'

Three-dimensional T1-weighted dark blood MRI sequencing determines suppression of the blood signal inside the vascular lumen, allowing visualization of the vascular wall. In the case of vasculitis, there is concentric thickening and avid contrast enhancement of the vascular wall ${ }^{1,2}$.

We report on a 35-year-old man with chronic meningitis and a ventriculoperitoneal shunt, presenting with sudden right hemiplegia. Cerebrospinal fluid analysis showed lymphocytic pleocytosis. Diffusion-weighted imaging revealed a recent ischemic lesion at the left nucleus-capsular level. Vascular wall imaging showed concentric thickening and avid contrast enhancement of the left M1 wall. Additionally, cervical spine MRI showed leptomeningeal thickening (Figure). Anatomopathological analysis revealed non-caseating granulomas.

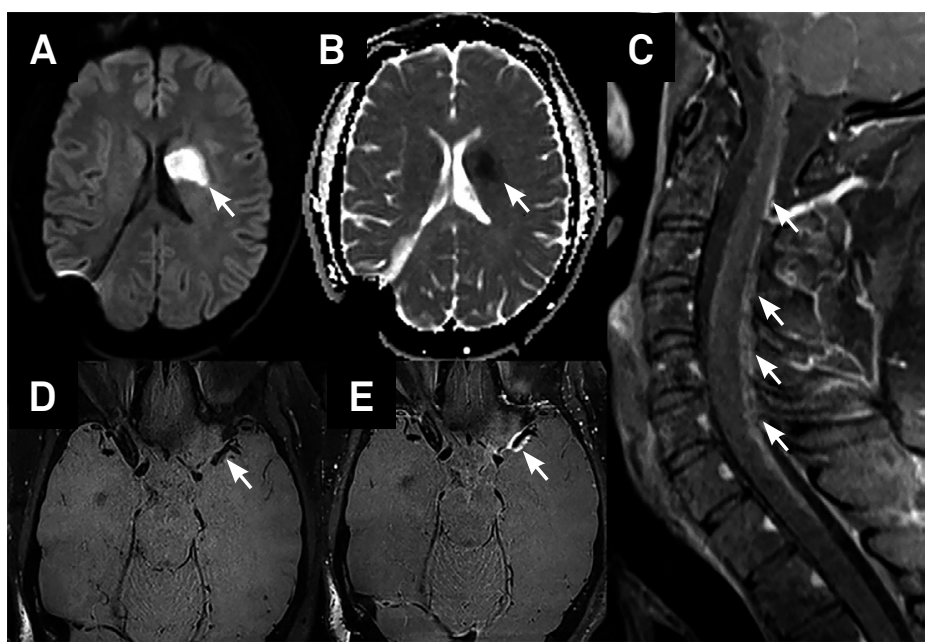

Figure. DWI (A) and ADC map (B) show ischemic insult at left nucleus-capsular level. Fat-suppressed post-contrast T1-weighted MRI (C) shows spinal leptomeningeal disease. Pre- (D) and post-gadolinium (E) 3D T1-weighted dark blood MRI reveals thickening and enhancement of left M1 wall, compatible with vasculitis.

\section{References}

1. Pinho MC, Hall JT, Cross JC, Shang T, Madhuranthakam AJ, Moore WA. Intracranial vessel wall MRI in Clinical practice: technical considerations, current and emerging applications, and clinical pearls and pitfalls. Radiographics. 2018 Apr; 8(2) 97-118. https://doi.org/10.3174/ng.9170237
2. Mandell DM, Mossa-Basha M, Qiao Y. Intracranial vessel wall MRI: Principles and expert consensus recommendations of the American Society of Neuroradiology. AJNR Am J Neuroradiol. 2017;38:218-29. https://doi.org/10.3174/ajnr.A4893

\footnotetext{
${ }^{1}$ Hospital das Américas, Departamento de Radiologia, Rio de Janeiro, RJ, Brasil; ${ }^{2}$ Hospital das Américas, Departamento de Neurologia, Rio de Janeiro, RJ, Brasil. Pedro Castro (iD) https://orcid.org/0000-0002-2956-1907; Roberto Santos (iD https://orcid.org/0000-0002-4681-8997; Dequitier Machado https://orcid.org/0000-0002-4874-8056; Felipe Schmidt (iD https://orcid.org/0000-0003-0491-5526; Denise Moreira (iD) https://orcid.org/0000-0001-8859-3039

Correspondence: Pedro Neves Paiva de Castro; Av.Jorge Curi, 550 - Barra da Tijuca; 22775-001 Rio de Janeiro RJ, Brasil; E-mail: pnpdc@yahoo.com.br Conflict of interest: There is no conflict of interest to declare.

Received 09 January 2019; Accepted 01 February 2019.
} 